\title{
The Globular Cluster Systems of NGC 1400 and NGC 1407
}

\author{
Kathryn M. Perrett, David A. Hanes ${ }^{1,2}$, Steven T. Butterworth ${ }^{1}$ and JJ Kavelaars ${ }^{2}$ \\ Department of Physics, Queen's University, Kingston, Ontario K7L 3N6, Canada \\ E-mail:perrett@astro.queensu.ca,hanes@astro.queensu.ca,bworth@astro.queensu.ca, kauelaar@astro.queensu.ca \\ Doug Geisler \\ National Optical Astronomy Observatories, Kitt Peak National Observatory \\ E-mail:doug@noao.edu \\ and \\ William E. Harris \\ Department of Physics and Astronomy, McMaster University, Hamilton, Ontario L8S 4M1, Canada \\ E-mail: harris@azalea.physics.mcmaster.ca
}

\begin{abstract}
The two brightest elliptical galaxies in the Eridanus A group, NGC 1400 and NGC 1407, have been observed in both the Washington $T_{1}$ and Kron-Cousins $I$ filters to obtain photometry of their globular cluster systems (GCSs). This group of galaxies is of particular interest due to its exceptionally high $M / L$ value, previously estimated at $\sim 3000 h$, making this cluster highly dark-matter-dominated (Gould 1993). NGC 1400's radial velocity $(549 \mathrm{~km} / \mathrm{s})$ is extremely low compared to that of the central galaxy of Eridanus A (NGC 1407 with $v_{\odot}=1766 \mathrm{~km} / \mathrm{s}$ ) and the other members of the system, suggesting that it is a foreground galaxy projected by chance onto the cluster. Using the shapes of the globular cluster luminosity functions, however, we derive distances of $17.6 \pm 3.1 \mathrm{Mpc}$ to NGC 1407 and 25.4 \pm 7.0 Mpc to NGC 1400. These results support earlier conclusions that NGC 1400 is at the distance of Eridanus A and therefore has a large peculiar velocity. Specific frequencies are also derived for these galaxies, yielding values of $S_{N}=4.0 \pm 1.3$ for NGC 1407 and $S_{N}=5.2 \pm 2.0$ for NGC 1400. In this and other respects, these two galaxies have GCSs which are consistent with those observed in other galaxies.
\end{abstract}

Subject headings: Globular cluster systems, galaxy clusters, dark matter

\footnotetext{
${ }^{1}$ Visiting Astronomer, Cerro Tololo Inter-American Observatory, National Optical Astronomy Observatories, operated by the Association of Universities for Research in Astronomy (AURA), Inc., under contract with the National Science Foundation (NSF).

${ }^{2}$ Visiting Astronomer, Canada-France-Hawaii Telescope, operated by the National Research Council of Canada, le Centre National de la Recherche Scientifique de France, and the University of Hawaii.
} 


\section{Introduction}

NGC 1400, a bright E0/S0 galaxy located in a large sub-condensation of the Eridanus cluster, has recently drawn attention to itself and its curious environment. The southern Eridanus group of galaxies extends between $3^{h} 15^{m} \lesssim$ R.A. $\lesssim 4^{h}$ and $-26^{\circ} \lesssim$ Dec. $\lesssim-15^{\circ}$, and exhibits a large degree of internal sub-clustering (see Figure 1 from Willmer et al. 1989). Its most concentrated clump of galaxies is Eridanus A $\left(3^{h} 40^{m},-19^{\circ}\right)$, which includes roughly 50 galaxies within a radius of $\sim 0.8$.

At an estimated distance of 16.4 Mpc (Tonry 1991), the large central E0 galaxy of the Eridanus A sub-cluster (NGC 1407) has a heliocentric velocity typical of the majority of the members of the group. NGC 1400, however, reveals an anomalously low redshift $(549 \mathrm{~km} / \mathrm{s})$ compared with those of its neighbouring galaxies, causing some uncertainty as to its true distance. Table 1 lists the members of Eridanus A for which heliocentric velocities have been determined. The mean radial velocity of these galaxies is $1643 \pm 95 \mathrm{~km} / \mathrm{s}$, or $1765 \pm 93 \mathrm{~km} / \mathrm{s}$ if NGC 1400 is not considered a member.

This straightforward question as to the true distance of a potential Eridanus A member has resulted in what may prove to be an extraordinary find: the sub-cluster has an abnormally high $M / L$ value. Of the 10 Eridanus A galaxies listed in Table 11, the two early-type galaxies NGC 1407 and NGC 1400 account for nearly $80 \%$ of the light. Adding the blue luminosities of the Eridanus galaxies within 0.8 of NGC 1407 (including NGC 1400) and calculating the mean of four virial mass estimators, Gould (1993) finds $M / L \sim 3125 h$.

This value is anomalously large; typically, groups or clusters of galaxies have $M / L$ values of several hundred, making Eridanus A one of the darkest clusters known. Considering only the virial theorem mass, the exclusion of NGC 1400 from the cluster mass calculation reduces $M / L$ by roughly a factor of 2 . Consequently, even if NGC 1400 has not virialized within the cluster, Eridanus A's $M / L$ is still excessively high.

If NGC 1400 is at the distance of Eridanus A, it may yet have originated from some other location, giving rise to a peculiar motion which is not due to the mass concentration of the sub-cluster. Since there are no other nearby mass concentrations large enough to account for NGC 1400's motion, this possibility is unlikely (see Gould 1993). Therefore, if NGC 1400 is at the distance of Eridanus A, it is likely to be dynamically associated with the sub-cluster. Is NGC 1400 indeed at the distance of Eridanus A, or is it instead a foreground galaxy mistakenly associated with the group due to projection effects?

Five independent studies have attempted to determine whether NGC 1400 is at the distance of Eridanus A. The results of four of these studies are summarized in Table 2 (see discussion in Gould 1993 and references therein). One additional piece of evidence presented by Gould is the globular cluster luminosity function (GCLF), credited as showing that NGC 1400 and NGC 1407 are at the same distance. However, these GCLFs are secondary results obtained by Tonry (1991) during the surface-brightnessfluctuation (SBF) analysis, and no rigorous investigation of the globular cluster systems of the two bright galaxies in Eridanus A has yet been performed.

We therefore elect to use the globular cluster luminosity functions of the two brightest ellipticals in Eridanus A, NGC 1400 and the central galaxy NGC 1407, to provide new estimates of their distances. In $\$ 2$ and $\$ 3$ we discuss the observations and data reduction, while in $\S$ we present the GCLFs and distance determinations. In addition, the inferred globular cluster radial profiles and scaled population sizes of these two galaxies are investigated in $\$ 5$ and $\$ 6$ respectively, in search of any signature of the peculiar environment in which they are found.

\section{Observations}

The data for this study of the globular cluster systems (GCSs) of NGC 1400 and NGC 1407 were acquired as part of two separate observing runs. The first set of observations was obtained on the nights of November 14 and 16, 1993, with the Cerro Tololo Inter-American Observatory (CTIO) 4-m telescope. Use of the Tek $2048 \times 2048$ CCD camera at prime focus with an image scale of $0 . \prime 46$ per pixel yielded an image size of $15^{\prime} .7 \times 15^{\prime} .7$. The readout noise was 6 electrons/pixel, and the gain was set to 3.2 electrons per ADU. A total of three images, each one including both NGC 1400 and NGC 1407, were taken using the Washington $T_{1}$ filter (Canterna 1976; Harris \& Canterna 1977). The seeing was mediocre at best, ranging from $1^{\prime \prime} 5$ to $2^{\prime \prime} .0$ over the two nights.

The second set of data was collected on January 2 and 3, 1995, with the Canada-France-Hawaii 3.6-m telescope (CFHT). The Loral3 detector $(2048 \times 2048)$ was used at a nominal gain of 1.45 electrons/ADU and with a readout noise of 9 electrons/pixel. This detector, mounted behind the re-imaging optics of the MOS instrument, yielded an image scale of $0{ }^{\prime \prime} 32$ pix and a frame size of $10^{\prime} .9 \times 10^{\prime} .9$. Six images, three each of NGC 1407 and NGC 1400, were obtained using the Kron-Cousins $I$-band filter. The seeing varied from 1 ." 1 to $1^{\prime \prime} .9$ over the two nights. A log of the observations is presented in Table 3. In addition to the program fields, standard star fields, dome flatfields and bias frames were also obtained.

\section{Data Reduction}

Preprocessing of the raw frames included bias subtraction and division by a mean dome flatfield exposure. Frames taken in comparable seeing were combined 
TABLE 1

Galaxies of Eridanus A with known velocities (Willmer et al. 1989).

\begin{tabular}{lccrc}
\hline \hline \multicolumn{1}{c}{ Galaxy } & $\begin{array}{c}\text { R.A. } \\
(1950)\end{array}$ & $\begin{array}{c}\text { Dec. } \\
(1950)\end{array}$ & $\begin{array}{c}v_{\odot} \\
(\mathrm{km} / \mathrm{s})\end{array}$ & $\mathrm{B}$ \\
\hline NGC 1383 & 33523 & -1830.1 & $1948 \pm 19$ & 14.0 \\
NGC 1390 & 33537 & -1910.3 & $1215 \pm 33$ & 14.6 \\
NGC 1393 & 33623 & -1835.4 & $2185 \pm 26$ & 13.9 \\
NGC 1400 & 33716 & -1851.0 & $549 \pm 21$ & 12.1 \\
IC 343 & 33752 & -1836.3 & $1869 \pm 30$ & 14.5 \\
NGC 1407 & 33757 & -1844.5 & $1766 \pm 21$ & 11.1 \\
ESO 548-G48 & 33804 & -1905.5 & $1817 \pm 27$ & 14.4 \\
IC 346 & 33929 & -1825.6 & $1897 \pm 47$ & 14.1 \\
ESO 548-G79 & 33941 & -1903.2 & $2053 \pm 31$ & 14.4 \\
ESO 549-G02 & 34043 & -1910.8 & $1134 \pm 36$ & 15.0 \\
\hline
\end{tabular}

a,b The galaxies in this study

TABle 2

Distance Measurements of NGC 1400 and NGC 1407 (Gould 1993).

\begin{tabular}{cccl}
\hline \hline Method & $\begin{array}{c}\text { NGC 1400 } \\
\text { Distance (Mpc) }\end{array}$ & $\begin{array}{c}\text { NGC 1407 } \\
\text { Distance (Mpc) }\end{array}$ & \multicolumn{1}{c}{ Reference } \\
\hline $\mathrm{SBF}$ & $16.3 \pm 1.0$ & $16.4 \pm 1.0$ & Tonry 1991 \\
$D_{n}-\sigma$ & $(24 \pm 1) h^{-1}$ & $(19 \pm 1) h^{-1}$ & Faber et al. 1989 \\
$\mathrm{X}-\mathrm{ray} / B$ & $(16 \pm 2) h^{-1}$ & $(18 \pm 1) h^{-1}$ & $\begin{array}{l}\text { Donnelly et al. 1990 } \\
(u-V) / V\end{array}$ \\
& $(40 \pm 2) h^{-1}$ & $(30 \pm 2) h^{-1}$ & $\begin{array}{l}\text { Sandage \& Visvanathan 1978; } \\
\text { Visvanathan \& Sandage 1977 }\end{array}$ \\
\hline
\end{tabular}


TABLE 3

Record of Observations.

\begin{tabular}{cccccccc}
\hline \hline Frame & Object(s) & Date & Start UT & Exp. (sec) & Filter & Airmass & Seeing $\left({ }^{\prime \prime}\right)$ \\
\hline $\mathrm{T}_{1 \mathrm{~A}}$ & $\mathrm{~N} 1400 / 1407$ & Nov. 14/93 & $08: 21$ & 600 & $T_{1}$ & 1.534 & 1.5 \\
$\mathrm{~T}_{1 \mathrm{~B}}$ & $\mathrm{~N} 1400 / 1407$ & Nov. 16/93 & $07: 28$ & 900 & $T_{1}$ & 1.294 & 1.9 \\
$\mathrm{~T}_{1 \mathrm{C}}$ & $\mathrm{N} 1400 / 1407$ & Nov. 16/93 & $07: 50$ & 900 & $T_{1}$ & 1.394 & 2.0 \\
$\mathrm{I}_{\mathrm{A}}$ & $\mathrm{N} 1407$ & Jan. 2/95 & $08: 48$ & 600 & $I$ & 1.447 & 1.4 \\
$\mathrm{I}_{\mathrm{B}}$ & $\mathrm{N} 1407$ & Jan. 2/95 & $09: 01$ & 600 & $I$ & 1.289 & 1.9 \\
$\mathrm{I}_{\mathrm{C}}$ & $\mathrm{N} 1407$ & Jan. 3/95 & $08: 01$ & 900 & $I$ & 1.345 & 1.1 \\
$\mathrm{I}_{\mathrm{D}}$ & $\mathrm{N} 1400$ & Jan. 2/95 & $08: 20$ & 600 & $I$ & 1.367 & 1.5 \\
$\mathrm{I}_{\mathrm{E}}$ & $\mathrm{N} 1400$ & Jan. 2/95 & $08: 33$ & 600 & $I$ & 1.397 & 1.5 \\
$\mathrm{I}_{\mathrm{F}}$ & $\mathrm{N} 1400$ & Jan. 3/95 & $07: 37$ & 900 & $I$ & 1.290 & 1.2 \\
\hline
\end{tabular}

to form composite images with improved signal-to-noise. The resultant composite images with substantially different seeing were reduced individually using the standard

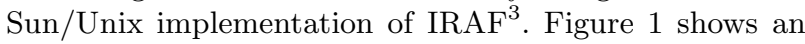
example of a preprocessed frame in the $T_{1}$ filter.

The data reduction was largely automated with the aid of an IRAF script and the DAOPHOT subroutines (Stetson 1987). A median filtering procedure was applied in order to create a model of the diffuse galaxy light which was then subtracted from the original image to better reveal the underlying globular cluster system. The fitting of a model stellar profile (a "point spread function" - PSF) was carried out upon the filtered image to yield the uncalibrated photometry list.

The fraction of objects successfully detected in each frame as a function of magnitude is known as the completeness function (Harris 1990). Using the IRAF ADDSTAR task, a total of $10^{3}$ artificial stars of known magnitudes were added to each frame in 10 trials, thus increasing the population of objects in an individual frame by roughly $20 \%$ per trial. In order to compensate for the variation in object detection with radial distance from the inner galaxy regions, separate completeness functions were determined for a series of annuli centred on the galaxy.

The uncertainties associated with the computed completeness function $(f)$ are derived assuming a binomial distribution:

$$
\sigma_{f}^{2} \approx \frac{f(1-f)}{n_{\text {add }}},
$$

where $n_{\text {add }}$ refers to the number of artificial stars added

\footnotetext{
${ }^{3}$ Image Reduction and Analysis Facility, distributed by the National Optical Astronomical Observatories, which is operated by AURA under contract with the NSF.
}

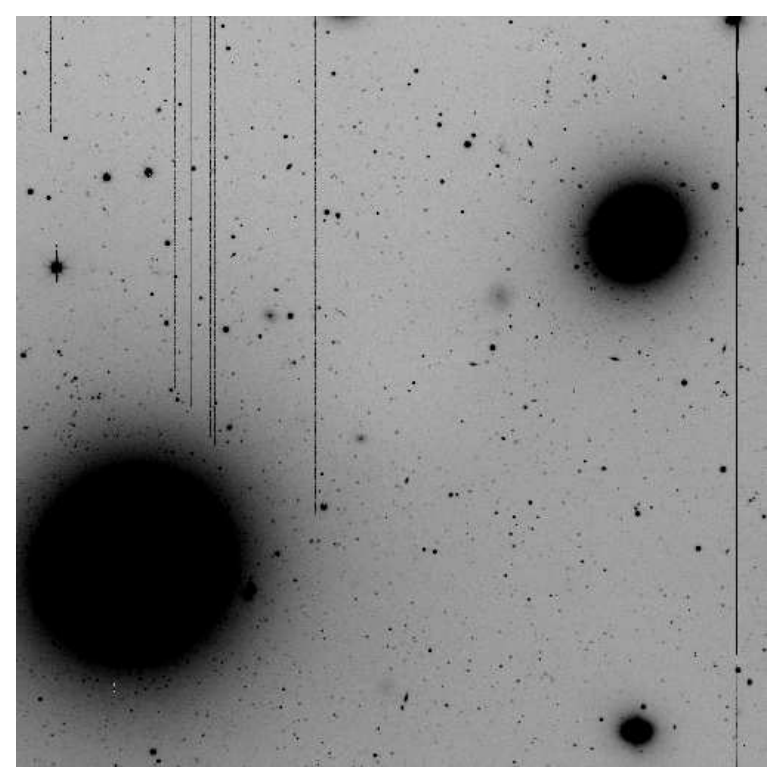

Fig. 1.- A preprocessed image of NGC 1400 (upper right) and NGC 1407 (lower left) in the $T_{1}$ filter from CTIO. The size of the field is $15^{\prime} .7 \times 15^{\prime} .7$ with north at the bottom and east at the left of the frame. 
to a given bin (Bolte 1989). The detection limits (50\% recovery levels) for the program frames are listed in Table 4. Using the known magnitudes of the artificial stars, we found that the systematic errors in the recovery process are negligible. An estimate of the internal uncertainty as derived from the scatter of the artificial stars was within $0.01 \lesssim \sigma \lesssim 0.05$ for all frames over the magnitude range of interest.

For galaxies as distant as NGC 1400 and NGC 1407, individual globular clusters are indistinguishable from stars under typical seeing conditions. A galaxy's GCS reveals itself as a statistical overabundance of faint, star-like features concentrated around the galaxy. An effective means of identifying non-stellar objects employs the CLASSIFY routine (Harris 1990). This routine calculates radial moments using weighted intensity sums around each object (see Harris et al. 1991 and references therein). We chose to use the effective radius parameter $r_{-2}$ as a discriminator:

$$
r_{-2}=\left(\frac{\sum\left[I_{j} /\left(r^{2}+0.5\right)\right]}{\sum I_{j}}\right)^{-1 / 2}
$$

where $r$ is the radial distance of the $j^{\text {th }}$ pixel from the object centroid, and $I_{j}$ is the intensity of the $j^{\text {th }}$ pixel after subtraction of the the local sky value. The summations were performed over those pixels within a maximum radius of 10 pixels which had intensities greater than the $3.5 \sigma$ detection threshold. For objects of a given magnitude, non-stellar objects such as faint background galaxies tend to exhibit more extended wings and faint cores, thus deviating towards larger values of $r_{-2}$. Upper limits were chosen such that roughly $95 \%$ of the artificial stars were correctly classified. In total, between $20 \%$ and $40 \%$ of the detected objects in each frame were identified as non-stellar and were removed from each photometry list.

Photometric calibrations were performed in the standard fashion. Fitting of transformation equations to the photometric magnitudes from short exposures of standard star fields (Geisler 1996, Porter observed during the same nights as the program fields yielded:

$$
\begin{gathered}
t_{1(a p)}-T_{1}=1.176+0.090\left(C-T_{1}\right)+0.048 X \\
i_{(a p)}-I=0.268+0.025(V-I)+0.114 X,
\end{gathered}
$$

where $t_{1(a p)}$ and $i_{(a p)}$ are instrumental magnitudes, and $T_{1}$ and $I$ are the known magnitudes of the standards. Since only $T_{1}$ and $I$ frames were obtained for the program data, the colour indices $C-T_{1}$ and $V-I$ were set to 1.5 and 1.0 respectively, typical values for globular clusters. The rms residuals for the transformations are \pm 0.05 in $T_{1}$ and \pm 0.04 in $I$.

\footnotetext{
${ }^{4}$ Unpublished: IRAF PHOTCAL standards database.
}

\section{Globular Cluster Luminosity Functions}

\subsection{Determining the GCLFs}

The observed globular cluster luminosity functions for NGC 1400 and NGC 1407 were obtained by binning the objects in the final photometry list into 0.4 mag intervals. We subtract the local sky LF (containing field objects and none of the GCS) from the observed LF (comprising both GCs and field objects) to reveal the GCLF for the target galaxy. No separate background frames were observed, hence the background density of objects was estimated from outer regions of the target galaxy frames where the GC density has dropped to zero (as determined from the radial profiles presented in the next section).

The galaxy LF and that for the background region were divided by their respective completeness corrections in order to compensate for increasing detection incompleteness at fainter magnitudes. Subtraction of the corrected background LF from the galaxy LF yields the globular cluster $\mathrm{LF}$ for each frame. The error bars on the luminosity distributions reflect the uncertainty on the inferred number of objects per magnitude bin:

$$
\sigma_{n}^{2} \approx\left[\frac{n_{\mathrm{obs}}}{f^{2}}+\frac{(1-f) n_{\mathrm{obs}}^{2}}{n_{\mathrm{add}} f^{3}}\right],
$$

(Bolte 1989). Since the completeness $(f)$ is the ratio of the observed number of objects $\left(n_{\mathrm{obs}}\right)$ to the number inferred in a magnitude bin $(n)$, Eq. 1 allows us to rearrange Eq. 3 in terms of the known quantities for each bin to obtain:

$$
\sigma_{n}^{2} \approx \frac{n}{f}\left(1+\sigma_{f}^{2} \frac{n}{f}\right) .
$$

Once the final cluster luminosity distributions of each data frame were calculated, the observed GCLFs corresponding to the same galaxy and filter were averaged, weighted by the uncertainty on the number counts. Unfortunately, for NGC 1400, the CTIO $T_{1}$ frames in best seeing were not long exposures (see Table 3), while the deeper exposures were in poorer seeing than enjoyed at CFHT. For these reasons the NGC 1400 GCLF was not well delineated in $T_{1}$; we chose to work with the $I$ observations exclusively. The final background-subtracted, completeness-corrected and averaged GCLFs in $T_{1}$ and $I$ for NGC 1407 and in $I$ for NGC 1400 are presented in Figures \&, 3 and 1 .

The globular cluster luminosity function is defined as the number of clusters per unit magnitude $(m)$ and can be described to first order by a Gaussian function:

$$
\phi(m)=A \exp \left(\frac{-\left(m-m^{0}\right)^{2}}{2 \sigma^{2}}\right),
$$

where $A$ is a normalization factor, $m^{0}$ represents the 
TABLE 4

Completeness Limits.

\begin{tabular}{cc}
\hline \hline Frame & $50 \%$ Limit \\
\hline $\mathrm{T}_{1 \mathrm{~A}}$ & $T_{1}=22.6$ \\
$\mathrm{~T}_{1 \mathrm{~B} / \mathrm{C}}$ & $T_{1}=22.4$ \\
$\mathrm{I}_{\mathrm{A}}$ & $I=21.6$ \\
$\mathrm{I}_{\mathrm{C}}$ & $I=22.2$ \\
$\mathrm{I}_{\mathrm{D} / \mathrm{E}}$ & $I=22.4$ \\
$\mathrm{I}_{\mathrm{F}}$ & $I=22.3$ \\
\hline
\end{tabular}

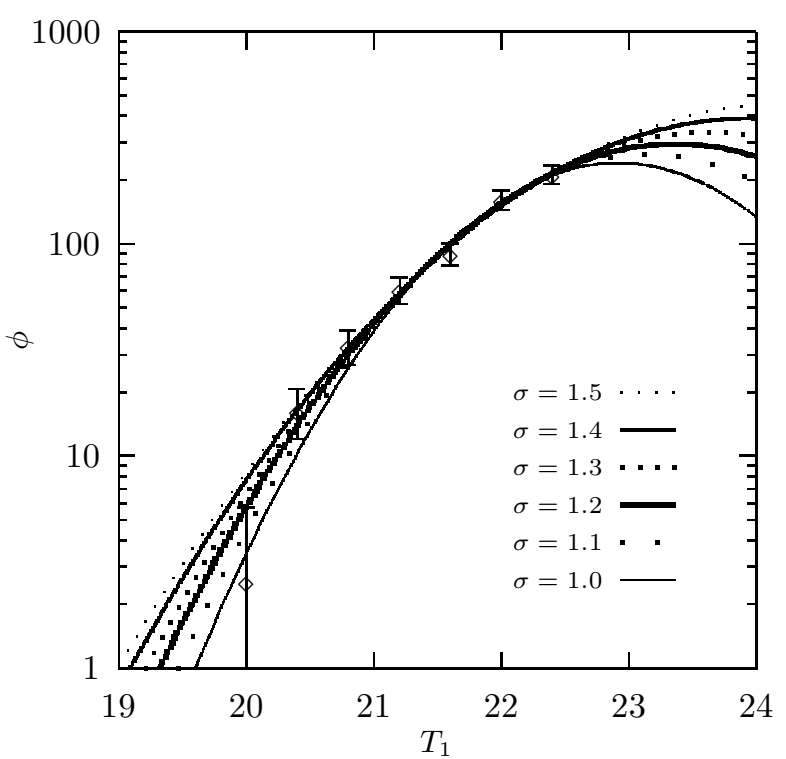

Fig. 2.- The GCLF of NGC 1407 in $T_{1}$ with Gaussian fits for a range of dispersions.

turnover (peak) magnitude and $\sigma$ is the dispersion (Hanes 1977).

To fit the Gaussian function of Eq. 月, a nonlinear leastsquares fitting procedure was applied. Since the data does not extend past the turnover magnitude, it was not possible to leave all three parameters $\left(A, m^{0}, \sigma\right)$ unconstrained during the fitting. Consequently, a series of values of $1.0 \lesssim \sigma \lesssim 1.5$ were adopted and held fixed while the scale factor and turnover magnitude were permitted to vary. These values of $\sigma$ span the range typically found for GCLFs (Harris 1991). The best-fit results are shown in Tables 5,6 and 7 . Note that the uncertainties quoted here reflect the formal errors associated with the least-squares fit.

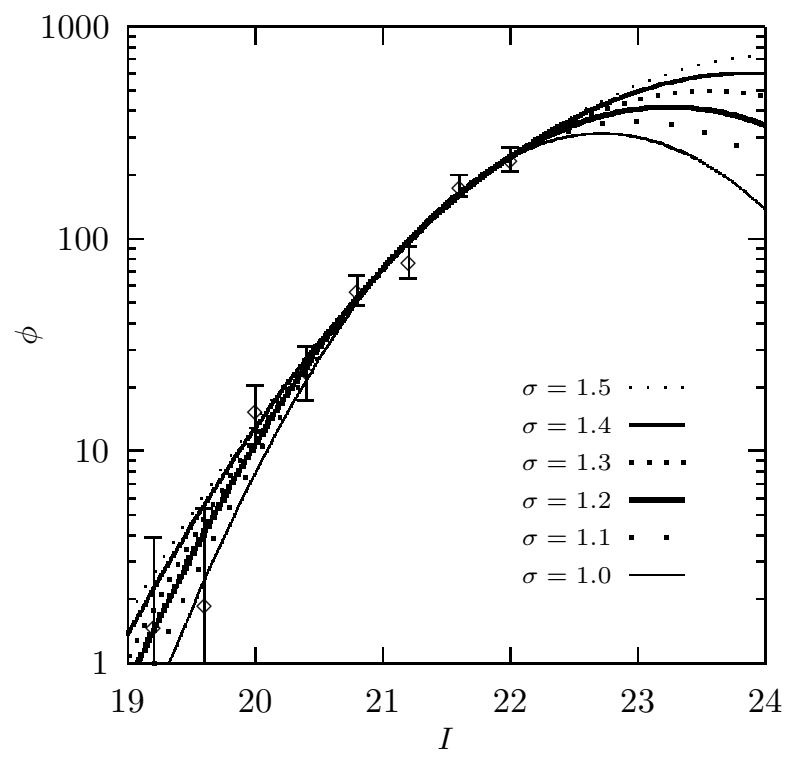

Fig. 3. - The GCLF of NGC 1407 in $I$ with Gaussian fits for a range of dispersions.

\subsection{Distance Determinations}

Comparing the observed brightness distributions for the globular cluster systems of NGC 1400 and NGC 1407 with those known from local calibrators, we can calculate distances to these galaxies. With an adopted absolute turnover magnitude of $M_{\mathrm{V}}^{0}=-7.27 \pm 0.23$ (a mean value for the 10 largest galaxies in Table 2 of Harris 1991) and assuming $\sigma=1.2$ for the GCLF shapes, we estimate distances of $17.6 \pm 3.1 \mathrm{Mpc}$ to NGC 1407 and 25.4 \pm 7.0 Mpc to NGC 1400 (Table 8). Note that the turnover magnitudes have been corrected for foreground absorption. For NGC 1407, we adopted galactic extinction corrections of $\mathrm{A}_{T_{1}}=0.11$ and $\mathrm{A}_{I}=0.06$, based on a value of $\mathrm{A}_{B}=0.17$ (RC3). NGC 1400's value of $\mathrm{A}_{B}=0.14$ yielded correc- 
TABLE 5

GCLF fit parameters for NGC 1407 in $T_{1}$.

\begin{tabular}{cccc}
\hline \hline$\sigma$ & $\mathrm{A}$ & $T_{1}^{0}$ & $\chi^{2}$ \\
\hline 1.0 & $241 \pm 15$ & $22.92 \pm 0.07$ & 5.2 \\
1.1 & $264 \pm 17$ & $23.13 \pm 0.07$ & 3.4 \\
1.2 & $296 \pm 22$ & $23.37 \pm 0.08$ & 2.8 \\
1.3 & $336 \pm 29$ & $23.64 \pm 0.09$ & 3.0 \\
1.4 & $389 \pm 41$ & $23.93 \pm 0.11$ & 3.6 \\
1.5 & $457 \pm 59$ & $24.24 \pm 0.13$ & 4.5 \\
\hline
\end{tabular}

TABle 6

GCLF fit parameters for NGC 1407 in $I$.

\begin{tabular}{cccc}
\hline \hline$\sigma$ & $\mathrm{A}$ & $I^{0}$ & $\chi^{2}$ \\
\hline 1.0 & $313 \pm 33$ & $22.72 \pm 0.11$ & 6.3 \\
1.1 & $358 \pm 45$ & $22.97 \pm 0.12$ & 4.9 \\
1.2 & $418 \pm 64$ & $23.25 \pm 0.15$ & 4.3 \\
1.3 & $498 \pm 92$ & $23.59 \pm 0.17$ & 4.3 \\
1.4 & $615 \pm 133$ & $23.89 \pm 0.20$ & 4.5 \\
1.5 & $749 \pm 194$ & $24.24 \pm 0.23$ & 5.1 \\
\hline
\end{tabular}

TABLE 7

GCLF fit parameters for NGC 1400 in $I$.

\begin{tabular}{cccc}
\hline \hline$\sigma$ & $\mathrm{A}$ & $I^{0}$ & $\chi^{2}$ \\
\hline 1.0 & $111 \pm 25$ & $23.22 \pm 0.21$ & 15.4 \\
1.1 & $130 \pm 37$ & $23.50 \pm 0.25$ & 15.6 \\
1.2 & $157 \pm 53$ & $23.80 \pm 0.30$ & 15.9 \\
1.3 & $194 \pm 79$ & $24.13 \pm 0.35$ & 16.1 \\
1.4 & $244 \pm 118$ & $24.49 \pm 0.41$ & 16.4 \\
1.5 & $316 \pm 178$ & $24.88 \pm 0.47$ & 16.7 \\
\hline
\end{tabular}




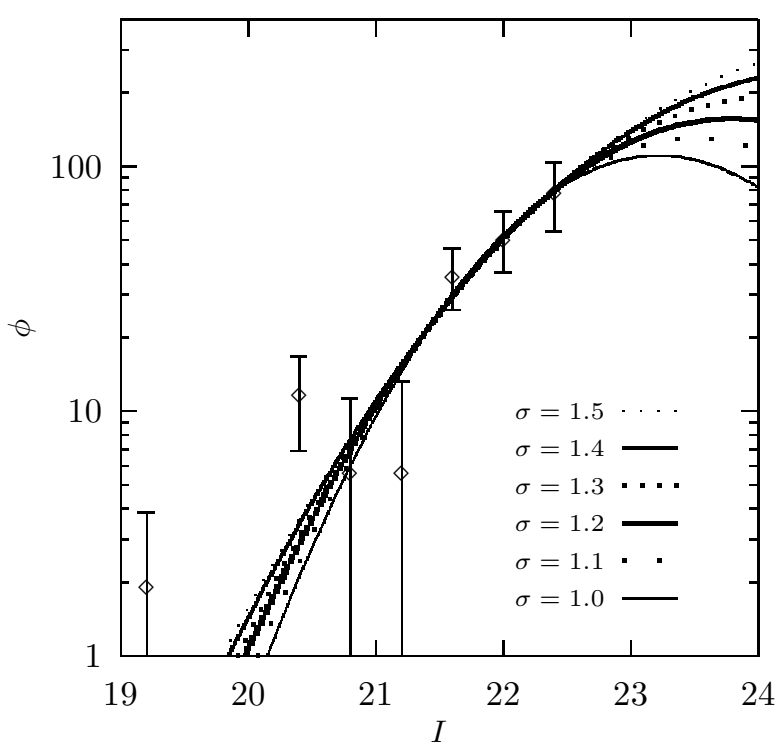

Fig. 4. - The GCLF of NGC 1400 in $I$ with Gaussian fits for a range of dispersions.

tions of $\mathrm{A}_{T_{1}}=0.09$ and $\mathrm{A}_{I}=0.06$ for this galaxy. The distances we have calculated in Table 8 are inconsistent with the hypothesis that NGC 1400 is a foreground galaxy roughly 3 times closer than NGC 1407, as implied by its anomalously low recessional velocity.

Further experimentation shows that the choice of dispersion $(\sigma)$ has little bearing on the relative distances obtained for these galaxies. The distance ratios fall within the range $d(N 1400) / d(N 1407)=1.31 \pm 0.04$ regardless of adopted $\sigma$, suggesting that NGC 1400 is in the background with respect to NGC 1407. Adopting the worst-case scenario in which $\sigma=1.5$ for NGC 1407 and $\sigma=1.0$ for NGC 1400 , we find that $d(N 1400) / d(N 1407)=0.63$, still placing NGC 1400 considerably further than the distance implied by its low recessional velocity. From this we may again conclude that, within the reasonable range of $\sigma$, our observations of the GCS of NGC 1400 are not consistent with it being a factor of 3 closer than NGC 1407.

If NGC 1400 were at a distance of $5.49 h^{-1} \mathrm{Mpc}$, the turnover magnitude of the globular cluster luminosity function would be expected to appear at $T_{1}^{0}=21.6$ and $I^{0}=21.0$, assuming $h=0.8$. With NGC 1400 GCS luminosity distribution data down to completeness limits of $T_{1}=22.6$ and $I=22.4$, our GCLFs should thus extend well beyond the turnover in such a case. Since the observed GCLFs for this galaxy do not show any evidence of having reached the turnover magnitude, this reaffirms our conclusion that NGC 1400 is not a foreground galaxy one third the distance of Eridanus A.

\section{Cluster Radial Profiles}

The surface density distribution of GCs plotted as a function of galactocentric radius reveals the projection of the spatial structure of the GCS. The annular bins used to construct the profiles were 75 pixels wide and concentric on the galaxy centroids. The number counts in each radial bin, corrected for completeness and divided by the total area of the annulus sampled, were taken to represent the surface density at the geometric mean annular radius $\left(r=\sqrt{r_{\text {in }} r_{\text {out }}}\right)$. Subtracting the local background density $\left(\sigma_{\mathrm{bgd}}\right)$, found by adopting the mean value of the surface density beyond the radius at which the radial profile is no longer decaying, we obtain the distribution of the GC population alone.

Non-linear least-squares fitting of the following relations were performed on the radial profiles:

$$
\begin{aligned}
\log \sigma_{\mathrm{GC}} & =a+b r^{1 / 4} \\
\log \sigma_{\mathrm{GC}} & =c+d \log r .
\end{aligned}
$$

The first model profile is a form of the empirical de Vaucouleurs law, and the second represents a scale-free power law (Harris 1986). The curves superimposed upon the radial profiles shown in Figures 1 to 8 represent the best fits to the data with the parameters and formal errors listed in Table 9. In all of the radial profile plots, we can clearly identify the presence of a centrally concentrated globular cluster system.

Surface intensity profiles of the galaxy halo light are also provided on the GC radial profile plots. These halo profiles were determined by fitting elliptical isophotes over the galaxy region, yielding the relative intensity of light as a function of radius. After subtracting the local sky level, the halo intensity was arbitrarily scaled to the amplitude of the GCS radial distribution for easy comparison.

\section{Specific Frequencies}

The specific frequency $\left(S_{N}\right)$ of a galaxy is defined as the number of globular clusters per unit halo light, normalized to an absolute magnitude of $M_{V}=15$ :

$$
S_{N} \equiv N_{t} \times 10^{0.4\left(M_{V}+15\right)}
$$

where the total population of GCs surrounding a galaxy is given by $N_{t}$ (Harris \& van den Bergh 1981). This parameter conveniently reflects the size of the GC population, independent of galaxy luminosity.

To calculate specific frequency we must first estimate the total number of clusters which make up the GCS of the galaxy. We use the radial density profiles from \$5 to count the total number of the clusters surrounding the galaxy down to the limiting magnitude $\left(N_{o b s}\right)$. We divide this 
TABLE 8

Galaxy distances assuming $\sigma=1.2$, corrected for absorption.

\begin{tabular}{ccccc}
\hline \hline Galaxy & $m^{\circ}$ & $V^{\circ}$ & $\left(V^{0}-M^{0}\right)$ & $d(\mathrm{Mpc})$ \\
\hline NGC 1407 & $T_{1}^{0}=23.26 \pm 0.08$ & $23.71 \pm 0.08$ & $30.98 \pm 0.31$ & $15.7\left(_{-2.1}^{+2.4}\right)$ \\
& $I^{0}=23.19 \pm 0.15$ & $24.19 \pm 0.15$ & $31.46 \pm 0.38$ & $19.5\left(_{-3.1}^{+3.7}\right)$ \\
NGC 1400 & $I^{0}=23.75 \pm 0.30$ & $24.75 \pm 0.30$ & $32.02 \pm 0.53$ & $25.4\left(_{-5.5}^{+7.0}\right)$ \\
\hline
\end{tabular}

TABLE 9

GC radial profile best-fit parameters.

\begin{tabular}{cccccc}
\hline \hline & & \multicolumn{2}{c}{$\log \sigma_{\mathrm{GC}}=a+b r^{1 / 4}$} & \multicolumn{2}{c}{$\log \sigma_{\mathrm{GC}}=c+d \log r$} \\
Galaxy & Filter & $a$ & $b$ & $c$ & $d$ \\
\hline \multirow{2}{*}{ NGC 1407 } & $T_{1}$ & $4.70 \pm 0.56$ & $-3.01 \pm 0.47$ & $1.77 \pm 0.12$ & $-2.23 \pm 0.34$ \\
& $I$ & $4.33 \pm 0.63$ & $-2.83 \pm 0.63$ & $1.46 \pm 0.11$ & $-1.74 \pm 0.38$ \\
NGC 1400 & $T_{1}$ & $4.62 \pm 1.27$ & $-3.71 \pm 1.22$ & $0.90 \pm 0.12$ & $-2.35 \pm 0.78$ \\
& $I$ & $3.79 \pm 0.41$ & $-2.81 \pm 0.46$ & $0.96 \pm 0.09$ & $-1.57 \pm 0.34$ \\
\hline
\end{tabular}

result by the fraction of the GCLF which was observed to infer the total number of clusters in the system. To define the shapes of the GCLFs, we adopt a Gaussian form (Eq. 5) assuming $\sigma=1.2$ and the best-fit parameters from Tables $\mathrm{O}, \mathrm{G}$ and $\mathrm{G}$.

Absolute $V$ magnitudes are also required in order to determine $S_{N}$. According to RC3, NGC 1407 has a corrected total blue magnitude $B_{\mathrm{T}}=10.5 \pm 0.2$ and colour $(B-V)_{\mathrm{T}}=0.97 \pm 0.01$, yielding $V_{\mathrm{T}}=9.5 \pm 0.2$. For NGC 1400, the RC3 values are $B_{\mathrm{T}}=11.87 \pm 0.13$ and $(B-V)_{\mathrm{T}}=0.92 \pm 0.01$, giving $V_{\mathrm{T}}=10.95 \pm 0.13$ for this galaxy. To translate $V_{\mathrm{T}}$ into absolute magnitude $M_{\mathrm{V}}$ we must adopt distances to the galaxies in question. For both galaxies, we assume a distance of $20.5 \pm 1.2 \mathrm{Mpc}$ based on the mean recessional velocity of the Eridanus A galaxies and $\mathrm{H}_{0}=80 \mathrm{~km} \mathrm{~s}^{-1} \mathrm{Mpc}^{-1}$. The results of the specific frequency calculations appear in Table 10. The specific frequencies calculated for NGC 1407 in the two filters are in agreement within their respective uncertainties, and we therefore adopt a mean value of $S_{N}=4.0 \pm 1.3$ for NGC 1407 along with that of $S_{N}=5.2 \pm 2.0$ for NGC 1400 .

\section{Discussion}

\subsection{The Question of Distance}

The primary motivation of this study was to determine whether NGC 1400 is at a distance comparable to that of NGC 1407 and the rest of Eridanus A, or if it is instead a foreground galaxy as implied by its anomalously low recessional velocity. The globular cluster luminosity functions are not consistent with the foreground placement of NGC 1400, which indicates that this galaxy has a large peculiar velocity with respect to Eridanus A, the origin of which remains unknown.

We may now add globular cluster systems to the evidence that NGC 1400 does indeed lie at roughly the distance of Eridanus A, and should be counted as a member of the cluster. The distances we have calculated assuming $\sigma=1.2$ for the GCLF are slightly higher but still within two standard deviations from those derived by Tonry (1991) of $16.3 \pm 1.0 \mathrm{Mpc}$ (NGC 1400) and $16.4 \pm 1.0 \mathrm{Mpc}$ (NGC 1407), and are well within the range of potential distances previously quoted in the literature (see Table 8 ).

\subsection{GCS Shapes and Specific Frequencies}

The spatial profiles of the globular cluster systems of NGC 1400 and NGC 1407 shown in $\$$ 国 seem rather unexceptional in shape. The radial distributions in Figures 5 and 6 reveal that the projected density of NGC 1407's GCS falls off with the same slope as the halo light intensity 
TABLE 10

Specific Frequencies

\begin{tabular}{cccccc}
\hline \hline Galaxy & Filter & $N_{\text {obs }}$ & $N_{t}$ & $M_{V}$ & $S_{N}$ \\
\hline NGC 1407 & $T_{1}$ & $629 \pm 76$ & $2296 \pm 279$ & $-22.06 \pm 0.33$ & $3.4 \pm 1.1$ \\
& $I$ & $556 \pm 61$ & $2985 \pm 327$ & $-22.06 \pm 0.33$ & $4.5 \pm 1.4$ \\
NGC 1400 & $I$ & $106 \pm 32$ & $922 \pm 280$ & $-20.61 \pm 0.26$ & $5.2 \pm 2.0$ \\
\hline
\end{tabular}

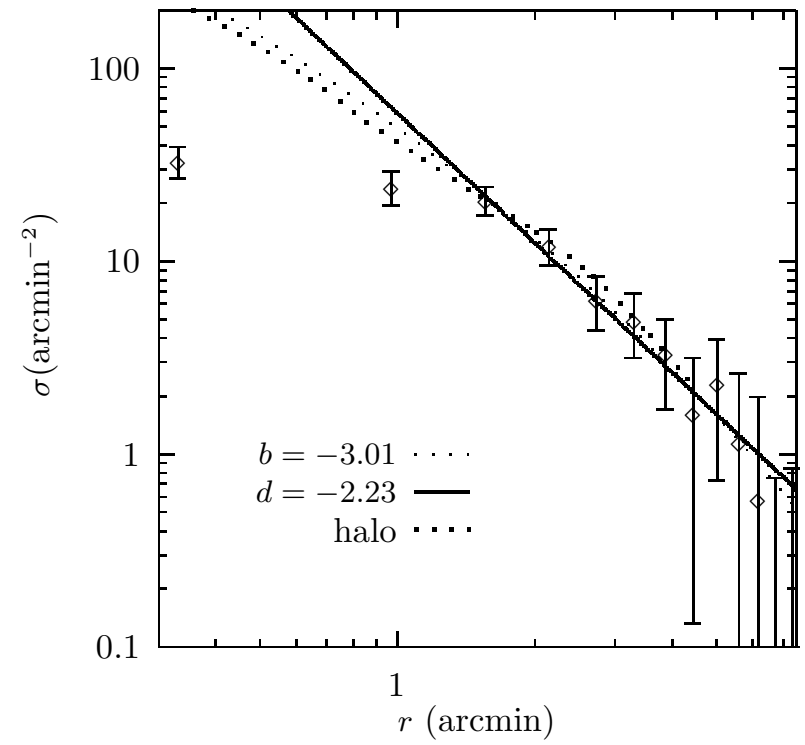

Fig. 5. - The background-subtracted radial density profile of the NGC 1407 GCS (in $T_{1}$ ) with de Vaucouleurs and scale-free power law fits provided (slopes given by $b$ and $d$ respectively). Also shown is the galaxy halo intensity profile. The background density level was found to be $\sigma_{\text {bgd }}=5.9 \pm 0.3$.

for this central elliptical galaxy of Eridanus A. Figures 7 and 8 indicate that the light profile for the NGC 1400 halo may be slightly steeper than the cluster density distribution, but this effect is not uncommon. It is somewhat unusual for GCS radial profiles to follow the halo light profile as closely as seen for NGC 1407; generally, the cluster surface density is more distended (Harris 1991). According to Merritt $(1983,1984)$, the cluster population and halo structure of a system dominated by dark matter will remain largely unchanged (to first order), not significantly altered by galaxy interaction processes. We are therefore likely seeing the cluster distributions as they were originally formed, with GCSs which are roughly as centrally

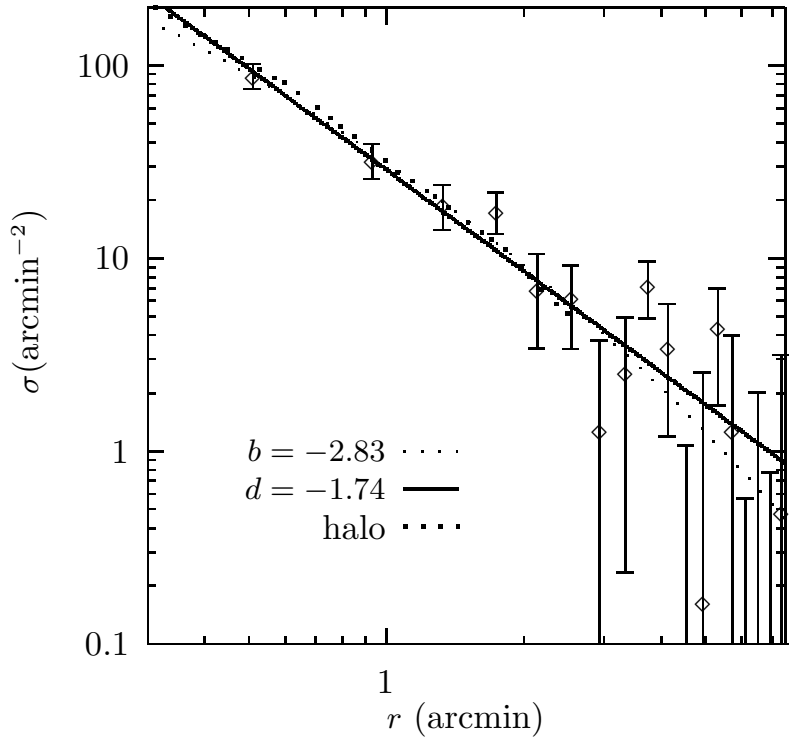

Fig. 6. - The NGC 1407 GCS radial density profile (in $I)$, as in Figure 5 . The background density was $\sigma_{\mathrm{bgd}}=$ $9.8 \pm 0.6$.

concentrated as the halo stars.

There exists an apparent relationship between the integrated $V$ magnitude of a galaxy and the shape of its GCS density profile (Harris 1986). The parameter generally used to describe the shape of the radial distribution is the slope of the logarithmic profile. This parameter was given the label " $d$ " in the power-law fitting of Section 5, but is commonly referred to as $\alpha$. A plot of the slope parameter as a function of galaxy magnitude is shown in Figure 9, with data and references presented in Table 11 . A linear fit to the data yields the relation:

$$
\alpha=-7.93-(0.29 \pm 0.03) M_{\mathrm{V}}^{\mathrm{T}}
$$

This is consistent with the relation as determined in Harris 1993 (see correction appearing in Kaisler et al. 1996).

The two galaxies in this study follow the overall 
TABLE 11

Correlation between radial density and galaxy luminosity.

\begin{tabular}{ccccc}
\hline \hline Galaxy & Type & $M_{\mathrm{V}}^{\mathrm{T}}$ & $\alpha$ & Source \\
\hline Milky Way & Sbc & -21.3 & $-2.5 \pm 0.26$ & H81, H76 \\
NGC 147 & dE & -15.0 & $-3.5 \pm 0.5$ & H86, HR79 \\
NGC 185 & dE & -15.2 & $-3.5 \pm 0.5$ & H86, HR79 \\
NGC 205 & dE & -16.5 & $-3.5 \pm 0.5$ & H86, HR79 \\
NGC 224 & Sb & -21.7 & $-2.4 \pm 0.4$ & H81, HR79 \\
NGC 524 & E/S0 & -22.1 & $-1.7 \pm 0.12$ & HH85 \\
NGC 1052 & E & -20.8 & $-2.26 \pm 0.27$ & HH85 \\
NGC 1374 & E & -19.8 & $-1.8 \pm 0.3$ & KKHRIQ96 \\
NGC 1379 & E & -20.0 & $-2.1 \pm 0.6$ & HH86b, KKHRIQ96 \\
NGC 1387 & S0 & -20.2 & $-2.2 \pm 0.3$ & HH86b, KKHRIQ96 \\
NGC 1399 & E & -21.2 & $-1.54 \pm 0.15$ & BHH91, KKHRIQ96 \\
NGC 1400 & E/S0 & -20.6 & $-1.96 \pm 0.56$ & This Paper \\
NGC 1404 & $\mathrm{E}$ & -20.8 & $-2.2 \pm 0.5$ & HH86b \\
NGC 1407 & $\mathrm{E}$ & -22.1 & $-1.99 \pm 0.36$ & This Paper \\
NGC 1427 & $\mathrm{E}$ & -20.0 & $-2.0 \pm 0.3$ & KKHRIQ96 \\
NGC 3115 & S0 & -21.1 & $-1.84 \pm 0.23$ & HH86a \\
NGC 3311 & cD & -22.4 & $-1.24 \pm 0.17$ & H86 \\
NGC 3842 & E & -23.2 & $-1.2 \pm 0.4$ & BH92 \\
NGC 4073 & cD & -23.1 & $-0.95 \pm 0.3$ & BH94 \\
NGC 4278 & E & -20.8 & $-2.26 \pm 0.23$ & Hv81 \\
NGC 4365 & E & -21.6 & $-1.15 \pm 0.25$ & HAPv91 \\
NGC 4472 & E & -22.9 & $-0.89 \pm 0.13$ & HAPv91 \\
NGC 4486 & E & -22.7 & $-1.55 \pm 0.08$ & H86 \\
NGC 4565 & Sb & -21.6 & $-2.33 \pm 0.61$ & FHPH95, H81, vH82 \\
NGC 4594 & Sa & -22.1 & $-2.0 \pm 0.2$ & HHH84 \\
NGC 4649 & E & -22.5 & $-1.08 \pm 0.10$ & HAPv91 \\
NGC 4944 & E & -21.04 & $-1.06 \pm 0.38$ & FHPH95 \\
NGC 5018 & E & -22.3 & $-1.3 \pm 0.4$ & HK96 \\
NGC 5128 & Ep & -22.0 & $-1.5 \pm 0.2$ & HHHC84 \\
NGC 5170 & Sb & -22.0 & $-1.69 \pm 0.25$ & FHHB90 \\
NGC 5481 & E & -20.2 & $-1.7 \pm 0.2$ & MR95 \\
NGC 5813 & E & -21.6 & $-2.18 \pm 0.33$ & Hv81 \\
NGC 7768 & cD & -22.9 & $-1.32 \pm 0.32$ & HPM95 \\
UGC 9799 & cD & -23.4 & $-1.39 \pm 0.30$ & HPM95 \\
UGC 9958 & cD & -23.4 & $-1.14 \pm 0.50$ & HPM95 \\
\hline & & & &
\end{tabular}




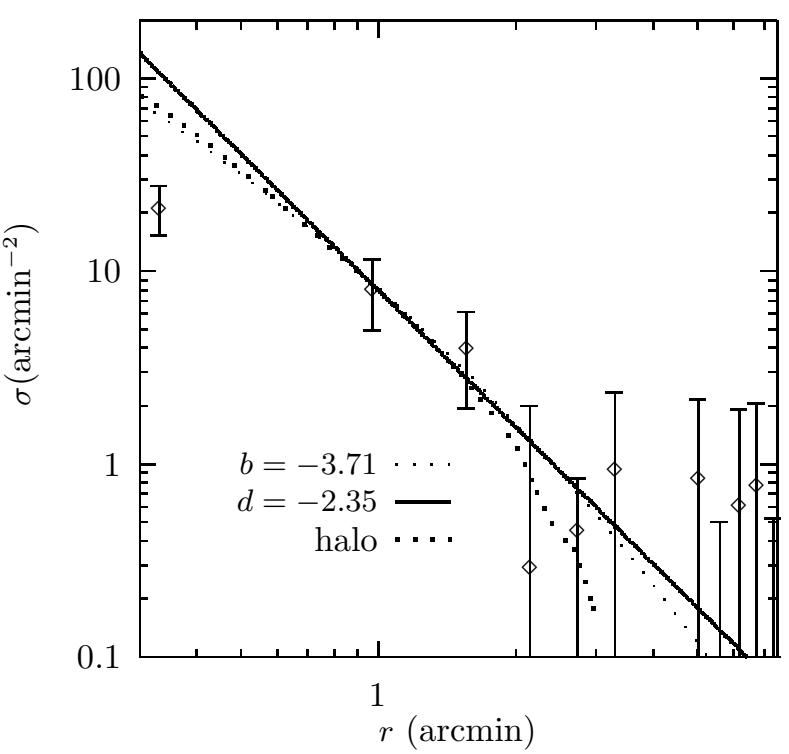

Fig. 7. - The NGC 1400 GCS radial density profile (in $T_{1}$ ), as in Figure $\mathrm{O}$. The background density was $\sigma_{\mathrm{bgd}}=$ $5.7 \pm 0.4$.

trend whereby higher-luminosity galaxies exhibit more extended globular cluster systems. The measured slopes, $\alpha(\mathrm{NGC} 1407)=-1.99 \pm 0.36$ and $\alpha(\mathrm{NGC1400})=-1.96 \pm$ 0.56 , compare reasonably well with the predicted values of $\alpha=-1.53$ and -1.95 respectively. If we do not include the data points for the dwarf ellipticals or the spiral galaxies, a linear regression yields

$$
\alpha=-7.55-(0.27 \pm 0.05) M_{\mathrm{V}}^{\mathrm{T}} .
$$

The omission does not significantly alter the relation; both of the linear fits above are shown in Figure 9.

The specific frequencies calculated for NGC $1407\left(S_{N}=\right.$ $4.0 \pm 1.3)$ and NGC $1400\left(S_{N}=5.2 \pm 2.0\right)$ are not unusual. Normal elliptical galaxies generally have $S_{N} \sim 2-5$, with variations from this range most likely attributable to differences in galaxy environment and GC formation mechanisms.

\subsection{GC Colours}

Although the $T_{1}$ and $I$ filters do not provide a sufficient baseline to obtain cluster metallicities, we can estimate mean colours for the globulars surrounding NGC 1407 and NGC 1400. Using the regions of overlap between the $T_{1}$ and $I$ images, we determine $T_{1}-I$ colours for the GCs detected in both filters. The colour distributions are shown in Figure 10; the lack of a well-defined peak in the NGC 1400 GCS colour distribution is likely due to the poor quality of the $T_{1}$ photometry for this galaxy, with its smaller cluster population. NGC 1407's cluster

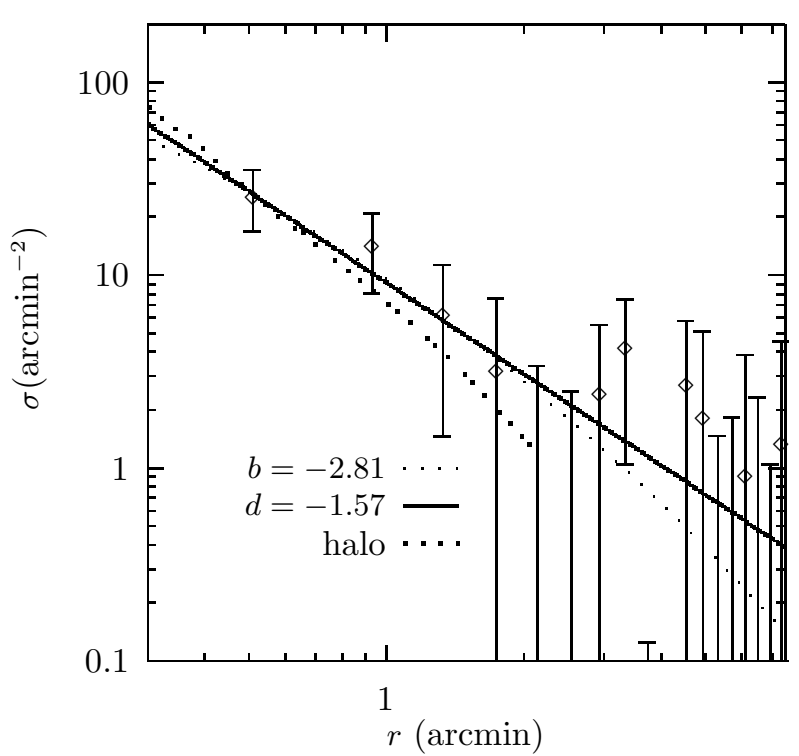

Fig. 8. - The NGC 1400 GCS radial density profile (in $I)$, as in Figure 6 . The background density was $\sigma_{\mathrm{bgd}}=$ $10.4 \pm 0.7$.

population is found to have a mean of $T_{1}-I=0.53$ and a median value of 0.54 , while for NGC 1400 we obtain mean and median colours of $T_{1}-I=0.54$ and 0.55 , respectively. These values are consistent with typical GC colours observed in other galaxies, a finding which confirms the identification of the GCSs.

\section{Conclusions}

The principal results of this study of the globular cluster systems of NGC 1400 and NGC 1407 can be summed up in the following points:

1. From the shapes of the globular cluster luminosity functions, we determine distances to these galaxies which place NGC 1400 at or beyond the distance of the Eridanus A group. This finding is in agreement with conclusions made using other methods previously cited in the literature.

2. The shapes of the GCS radial density profiles and the specific frequencies of the two systems reveal no obvious abnormalities. This implies that, if Eridanus $\mathrm{A}$ is as dominated by dark matter as its estimated $M / L$ value indicates, no anomalies are evident from the GC spatial distributions and population sizes of its two largest galaxies.

With a distance comparable to that of NGC 1407, NGC 1400 must have a high peculiar velocity in order to account for its exceptionally low radial velocity. Gould 


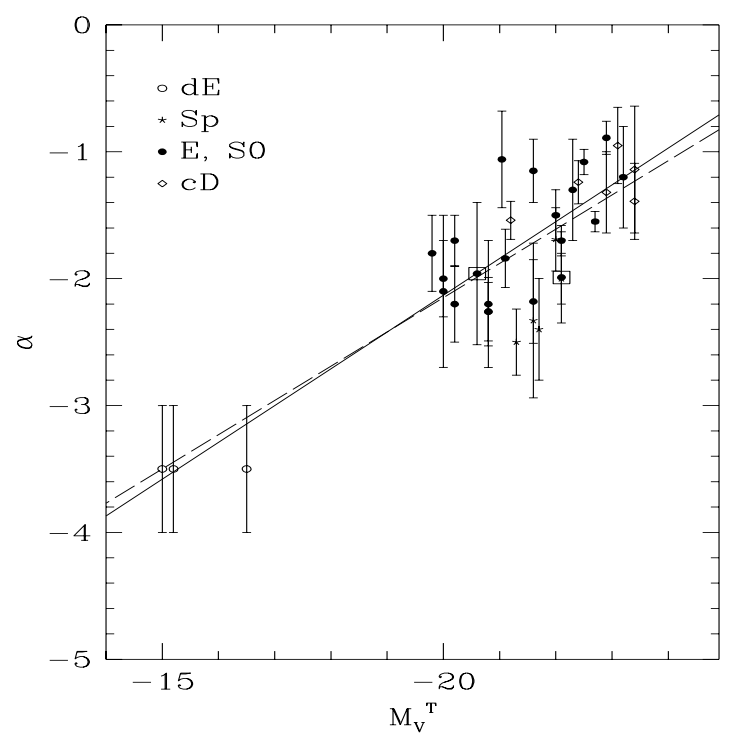

Fig. 9.- The relationship between slope of the GCS radial distribution and total galaxy $\mathrm{V}$ magnitude. The solid line represents a linear fit to the data including all data points (Eq. 8a), and the dashed line indicates the fit if one does not include dwarf or spiral galaxies in the sample (Eq. 8b). The data points enclosed by large open squares highlight the values obtained for NGC 1400 and NGC 1407 in the present study.

(1993) demonstrates that if the distance of NGC 1400 is consistent with that of Eridanus A, it must be bound to the sub-cluster due to a lack of other nearby mass concentrations large enough to generate its high peculiar motion. The exact cause for the large peculiar velocity remains as yet unknown. It is possible that NGC 1400 has a large component of its velocity moving it towards the core of Eridanus A (ie: it has a large transverse velocity), reducing its net radial velocity. Perhaps the velocity dispersion of the cluster has been severely underestimated since we only have velocity data for 10 of the 50 or so members, and that by some coincidence the other galaxies in the sample have significant velocity components perpendicular to our line of sight. If this is the case, NGC 1400's high peculiar velocity might not be particularly anomalous.

The second enigma surrounding the Eridanus A subcluster is its abnormally high $M / L$ ratio. It is possible that it is merely a dark cluster - many such clusters could exist which have so far avoided detection. More expansive surveys at higher limiting magnitudes in combination with reliable cluster-finding algorithms may reveal the presence of more dark clusters. The question remains: if indeed this cluster contains a great deal of dark matter, where did it come from, and why is NGC 1400 the only member (so

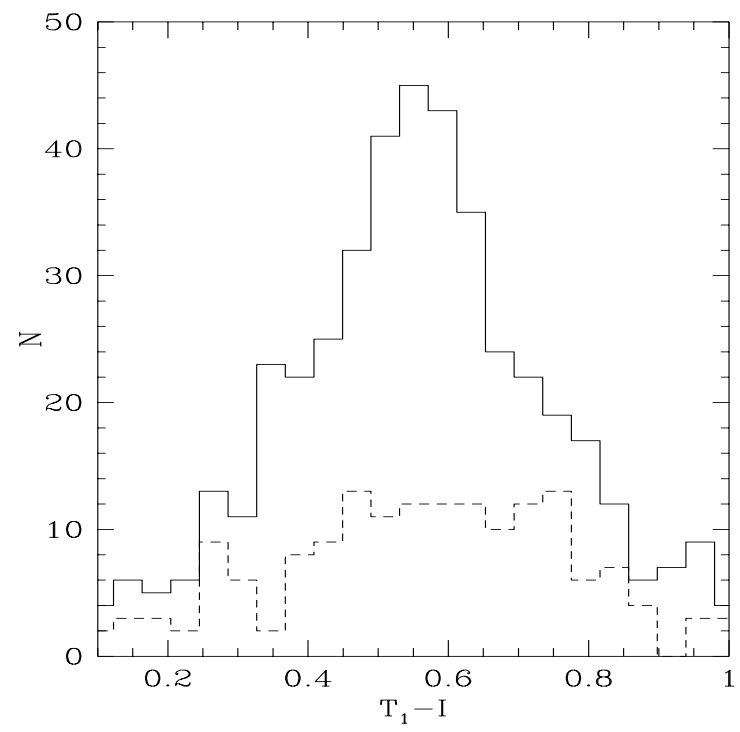

Fig. 10.- Colour distribution for the globular clusters surrounding NGC 1407 (solid line) and NGC 1400 (dashed line).

far) to show such a high peculiar velocity? Furthermore, why is there no evidence of strange effects on the radial distributions and population sizes of the member galaxy GCSs, for example, given this unusual environment?

A more extensive analysis of the GCS of the two Eridanus A galaxies NGC 1407 and NGC 1400 could be provided by additional deeper multicolour photometry as well as spectroscopic observations. This data may contribute to a better understanding of the nature of the galaxies and their environment. There is an obvious lack of redshift measurements for the majority of the Eridanus A galaxies (see Table 4 of Ferguson \& Sandage (1990) for the complete membership list). This makes it difficult to determine an accurate $M / L$ ratio for the cluster, as well as to derive any conclusions regarding the dynamical processes at work within Eridanus A. A more complete database of member galaxy velocities may shed some light on this dark cluster.

The authors wish to extend their gratitude to the kind and helpful staff at CTIO and CFHT. This work was supported by the Natural Sciences and Engineering Research Council of Canada in the form of operating grants (DAH and WEH) and a PGS A scholarship (KMP).

\section{REFERENCES}

Bolte, M. 1989, ApJ, 341, 168.

Bridges, T. J., and Hanes, D. A. 1994, ApJ, 431, 625 
(BH94).

Bridges, T. J., Hanes, D. A., and Harris, W. E. 1991, AJ, 101, 469 (BHH91).

Butterworth, S. T., and Harris, W. E. 1992, AJ, 103, 1828 (BH92).

Canterna, R. 1976, AJ, 81, 228.

de Vaucouleurs, G., de Vaucouleurs, A., Corwin, H. G., Buta, R. J., Paturel, G., and Fouqué, P. 1991, Third Reference Catalogue of Bright Galaxies (New York: Springer-Verlag), 284 (RC3).

Donnelly, R. H., Faber, S. M., and O'Connell, R. M. 1990, ApJ, 354, 52.

Faber, S. M., Wegner, G., Burstein, D., Davies, R. L., Dressler, A., Lynden-Bell, D., and Terlevich, R. J. 1989, ApJS, 69, 763.

Ferguson, H. C., and Sandage, A. 1990, AJ, 100, 1.

Fischer, P., Hesser, J. E., Harris, H. C., and Bothun, G. D. 1990, PASP, 102, 5 (FHHB90).

Fleming, D. E. B., Harris, W. E., Pritchet, C. J., Hanes, D. A. 1995, AJ, 109, 1044 (FHPH95).

Geisler, D., 1996, AJ, 111, 480.

Gould, A. 1993, ApJ, 403, 37.

Hanes, D. A. 1977, MNRAS, 180, 309.

Hanes, D. A., and Harris, W. E. 1986a, ApJ, 304, 599 (HH86a).

Hanes, D. A., and Harris, W. E. 1986b, ApJ, 309, 564 (HH86b).

Harris, G. L. H., Hesser, J. E., Harris, H. C., and Curry, P. 1984, ApJ, 287, 175 (HHHC84).

Harris, H. C., and Canterna, R. 1977, AJ, 82, 798.

Harris, W. E. 1976, AJ, 81, 1095 (H76).

Harris, W. E. 1981, ApJ, 251, 497 (H81).

Harris, W. E. 1986, AJ, 91, 822 (H86).

Harris, W. E. 1990, PASP, 102, 949.

Harris, W. E. 1991, ARA\&A, 29, 543.

Harris, W. E. 1993, The Globular Cluster-Galaxy Connection, ed. G. E. Smith \& J. P. Brodie (ASP Conf. Ser. 43), 472.

Harris, W. E., Allwright, J. W. B., Pritchet, C. J., and van den Bergh, S. 1991, ApJS, 76, 115 (HAPv91).

Harris, W. E., and Hanes, D. A. 1985, ApJ, 291, 147 (HH85).

Harris, W. E., Harris, H. C., and Harris, G. L. H. 1984, AJ, 89, 216 (HHH84).

Harris, W. E., Pritchet, C. J., and McClure, R. D. 1995, ApJ, 441, 120 (HPM95).
Harris, W. E., and Racine, R. 1979, ARA\&A, 17, 241 (HR79).

Harris, W. E., and van den Bergh, S. 1981, AJ, 86, 1627 (Hv81).

Hilker, M., Kissler-Patig, M. 1996, A\&A, Preprint (HK96).

Kaisler, D., Harris, W. E., Crabtree, D. R., and Richer, H. B. 1996, Preprint.

Kissler-Patig, M., Kohle, S., Hilker, M., Richtler, T., Infante, L., and Quintana, H. 1996, A\&A, Preprint (KKHRIQ96).

Madejsky, R., and Rabolli, M. 1995, A\&A, 297, 660 (MR95)

Merritt, D. 1983, ApJ, 26424.

Merritt, D. 1984, ApJ, 27626.

Sandage, A., and Visvanathan, N. 1978, ApJ, 223, 707.

Stetson, P. B. 1987, PASP, 99, 191.

Tonry, J. L. 1991, ApJ, 373, 1.

van den Bergh, S., and Harris, W. E. 1982, AJ, 87, 494 (vH82).

Visvanathan, N., and Sandage, A. 1977, ApJ, 216, 214.

Willmer, C. N. A., Focardi, P., da Costa, L. N., and Pellegrini, P. S. 1989, AJ, 98, 1531.

This 2-column preprint was prepared with the AAS LATEX macros $\mathrm{v} 4.0$. 